\title{
125ppm Resolution and 120dB Dynamic Range Nanoparticle Chemiresistor Array Readout Circuit
}

\author{
Xiaoyi Mu, Daniel Rairigh, Andrew J. Mason, Senior Member, IEEE \\ Electrical and Computer Engineering, Michigan State Univ., East Lansing, MI, USA
}

\begin{abstract}
Nanoparticle coated chemiresistor (CR) arrays enable highly sensitive vapor detection in systems such as a micro gas chromatograph or an electronic nose. However, they suffer shortcomings such as a small response compared to a large baseline value, large baseline variation across devices, and significant baseline drift over time. This paper describes a new high-resolution $C R$ array readout circuit with adaptive baseline control. The 8-channel readout circuit occupies $2.2 \mathrm{~mm}$ $x \quad 2.2 \mathrm{~mm}$ in $0.5 \mu \mathrm{m}$ CMOS technology, consuming $66 \mu \mathrm{W}$ per channel from a $3.3 \mathrm{~V}$ power supply. It achieves a worst-case resolution of $125 \mathrm{ppm}$ over a baseline resistance of $60 \mathrm{k} \Omega$ to $10 \mathrm{M} \Omega$, equivalent to $120 \mathrm{~dB}$ dynamic range.
\end{abstract}

\section{INTRODUCTION}

Chemiresistor (CR) sensors are able to detect, classify and quantify volatile organic compounds and have wide application in civilian and military environmental monitoring. For instance, highly sensitive vapor sensors using thiolate-monolayer-protected gold nanoparticle (MPN) coatings can provide selective determinations of a wide range of vapors by virtue of the pattern derived from the collective responses of a sensor array. MPN-based CRs have been reported for the determination of indoor air contaminants and environmental tobacco smoke markers [14]. However, to fully exploit the high sensitivity of MPNcoated CR sensors, a readout circuit is needed that addresses the shortcomings of chemiresistors, particularly their large baseline resistance $\left(\mathrm{R}_{\mathrm{b}}\right)$, up to $10^{6}$ times the actual sensor response $(\Delta R)$; the wide variability of $R_{b}$, up to two orders of magnitude across sensor elements; and significant drift over time at rates varying with each sensor element.

To date, many of the interface circuits developed for CRs have not provided the tracking or cancellation of baseline values that are needed to permit high resolution readout of the actual response portion of the sensor's resistance. Resistance-to-frequency interface circuits [5-6] and logarithmic converters [7] are well suited for digitizing a wide range of resistances but have limited resolution because they digitize the large baseline value along with the small sensor response. Higher resolution was achieved using a passivated (non-sensing) element as a reference for baseline cancellation [8]. However, this approach has limited utility because fabricating two sensors with identical initial baseline and drift characteristics is nearly impossible

This work is supported by the Science and Technology Directorate of the U.S. Department of Homeland Security (06-G-024). with many sensor technologies. Alternatively, baseline cancellation using an op-amp with resistive feedback and a power gain stage has been reported to provide a resolution $\left(\Delta \mathrm{R} / \mathrm{R}_{\mathrm{b}}\right)$ of about $200 \mathrm{ppm}$ [9]. However, it does not provide drift tracking. A baseline cancellation approach in the current domain [10] provided $160 \mathrm{~dB}$ dynamic range but achieved a resolution of only $1000 \mathrm{ppm}$.

Our group has previously reported an approach in which a subtraction circuit eliminates multiple noise sources without feedback resistors or a power gain stage, thus reducing area and power consumption [11]. This paper reports a new generation of this baseline tracking and cancellation approach that achieves a higher resolution and wider dynamic range while implementing all of the necessary circuitry in a single chip. The new circuit integrates a widerange programmable current bias, an 8-bit digital to analog converter (DAC) for analog memory (AM), and a digitallycontrolled 8-channel readout block to provide high resolution readout with adaptive dynamic range that enables tracking baseline resistance drift. The chip also supports post-CMOS fabrication of an MPN sensor array directly on the surface of the chip to eliminate wiring noise. Section II describes CR readout requirements and outlines our approach to achieve high resolution. Section III describes the circuit implementation, and testing results are presented in Section IV. Finally, a conclusion is provided in Section V.

\section{REQUIREMENTS AND APPROACH FOR HIGH SENSITIVITY}

The normalized resistance change $\left(\Delta R / R_{b}\right)$ of an MPNcoated $\mathrm{CR}$ sensor is directly related to the concentration of target vapors. However, the sensor response portion of the $\mathrm{CR}, \Delta \mathrm{R}$, is small and buried within the large total value $\Delta \mathrm{R}$ $+R_{b}$. Furthermore, $R_{b}$ varies widely from sensor to sensor. To acquire $\Delta R / R_{b}$ with high resolution, the readout circuit must both overcome the large variation of $R_{b}$ and precisely measure small $\Delta R$ values. In prior work [11], we introduced a baseline resistance cancelation approach that subtracts $R_{b}$ from the total $C R$ resistance and digitizes the $\Delta \mathrm{R}$ portion. The resolution of $\Delta R$ is limited by how accurately $R_{b}$ is measured and subtracted. Because $R_{b}$ is subject to significant drift, it is important to track $R_{b}$ closely over the wide dynamic range typical to $C R$ sensors. Canceling $R_{b}$ in the analog domain and amplifying only $\Delta \mathrm{R}$ significantly improves the overall signal to noise ratio (SNR).

To meet these requirements, the architecture for the CR 


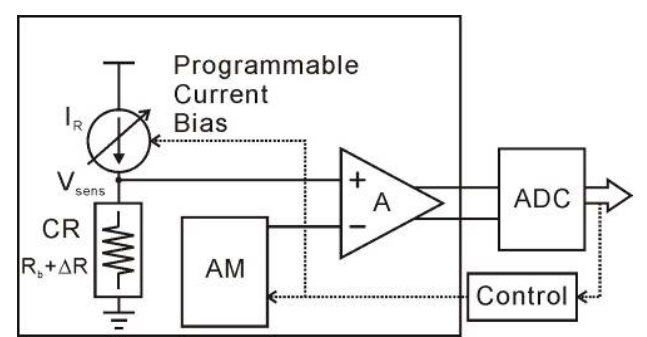

Fig. 1. Single channel architecture for $\mathrm{CR}$ baseline tracking and cancellation.

readout circuit (one channel) is shown in Fig. 1. The CR sensor is stimulated by a constant bias current $I_{R}$, and the sensor voltage response is

$$
V_{\text {sens }}=I_{R} R_{\text {sens }}
$$

where $R_{\text {sens }}=\Delta R+R_{b}$. The circuit works in two phases. In the idle phase $(\Delta \mathrm{R}=0)$, a calibration is performed to determine the bias current such that $V_{\text {sen } 1}=I_{R} \cdot R_{b}$ is between $V_{\text {min }}$ and $V_{\text {max }}$. Because $R_{b}$ can vary by more than two orders of magnitude, this large resistance range needs to be mapped into a small voltage range by adjusting the programmable current bias. The determined value of $\mathrm{V}_{\text {sens } 1}$ is then stored on the analog memory (AM) to end the idle (calibration) phase.

During the response phase, $V_{\text {sen } 2}=I_{R} \cdot\left(R_{b}+\Delta R\right)$ is input to the subtraction and gain block where the baseline value previously stored on the $\mathrm{AM}\left(\mathrm{V}_{\text {sens1 }}\right)$ is subtracted from the input $\left(\mathrm{V}_{\text {sens } 2}\right)$ and the difference is amplified by a programmable gain. After these two phases, $V_{\text {sens1 }}$ and $\left(\mathrm{V}_{\text {sens2 }}-\mathrm{V}_{\text {sens1 }}\right)$ are obtained, corresponding to $\mathrm{R}_{\mathrm{b}}$ and $\Delta \mathrm{R}$. With subsequent $\mathrm{A} / \mathrm{D}$ conversion and offline calculations, $\Delta \mathrm{R} / \mathrm{R}_{\mathrm{b}}$ can readily be obtained from $\left(\mathrm{V}_{\text {sens } 2}-\mathrm{V}_{\text {sens1 } 1}\right) / \mathrm{V}_{\text {sens } 1}$. Because the $\Delta \mathrm{R}$ signal is very small and susceptible to noise, the subtraction and gain block is fully differential to minimize common mode noise. Input offsets of the subtraction and gain block, as well as offsets caused by the analog memory's finite bit resolution, are eliminated by this sample and subtract process.

\section{CIRCUIT IMPLEMENTATION}

The CR baseline tracking and cancellation approach first described in [11] has been significantly expanded and improved in the 8-channel circuit shown in Fig. 2. The new, fully integrated system contains a subtraction and gain block, a wide-range programmable exponential current bias, an 8-bit DAC for the AM, a digital communication and control circuit, and surface contacts to support an on-chip array of MPN-coated CR sensors.

\section{A. Programmable Exponential Current Bias}

For the MPN-coated CR sensors targeted by this system, $\mathrm{R}_{\mathrm{b}}$ has been observed to vary from $100 \mathrm{k} \Omega$ to $10 \mathrm{M} \Omega$. To extract the same level of $\Delta R / R_{b}$ resolution for all values of $R_{b}$, a programmable current bias is essential for mapping baseline resistance into a narrow output voltage range, $V_{b}$. Assuming that the range of $\mathrm{V}_{\mathrm{b}}$ is held within $\mathrm{V}_{\min }$ to $\mathrm{V}_{\max }$ by

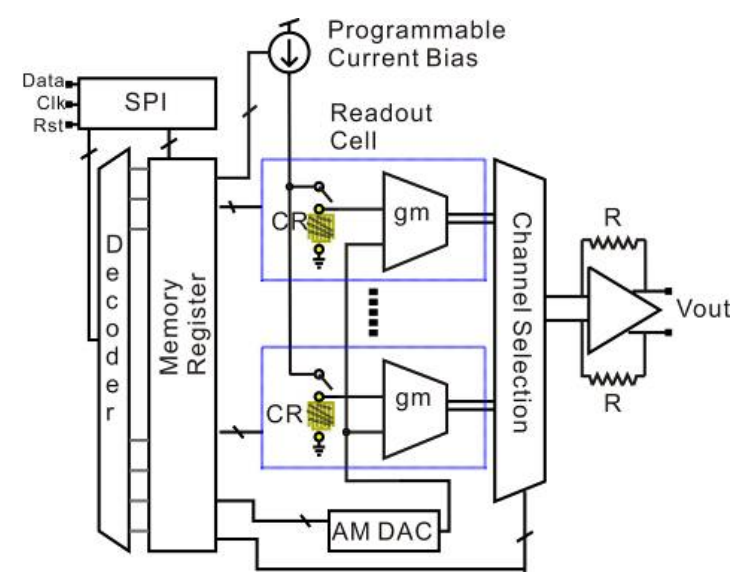

Fig. 2. Simplified schematic of the 8-channel CR readout circuit that integrates subtraction and gain cells, an analog memory DAC, a programmable current bias and a digital control block.

the current source and that the discrete programmable current is $\mathrm{I}[\mathrm{n}]$, where $n$ is number of current values available, the current values $I[n]$ provided by the system must be set such that

$$
\frac{V_{\max }}{I[n]} \leq R_{b} \leq \frac{V_{\min }}{I[n]}
$$

In other words, for all values of $R_{b}$, a current $I[n]$ should be available such that the conditions in (2) are met. To ensure all possible $R_{b}$ values generate a voltage between $V_{\text {min }}$ and $\mathrm{V}_{\max }$, the $\mathrm{V}_{\mathrm{b}}$ steps for each $\mathrm{I}[\mathrm{n}]$ must overlap. This can be expressed mathematically as

$$
\frac{V_{\min }}{I[n]} \leq \frac{V_{\max }}{I[n-1]}, \frac{V_{\max }}{I[n+1]} \leq \frac{V_{\min }}{I[n]}
$$

When the boundary values of adjacent steps are set equal to each other, (3) becomes

$$
\frac{I[n]}{I[n-1]}=\frac{I[n+1]}{I[n]}
$$

This relationship indicates that an exponential current bias would best span the required range with the fewest steps. The current bias circuit shown in Fig. 3 was designed to achieve this goal. Transistors M6 to M27 have a large width and length to minimize flicker noise, reduce errors due to process mismatch, and bolster output resistance. The current variations caused by variable $\mathrm{V}_{\text {sens }}$ are negligible because M6 to M27 have a large gate length and their drain voltages are narrowly held between $\mathrm{V}_{\max }$ and $\mathrm{V}_{\text {min }}$. The lower five

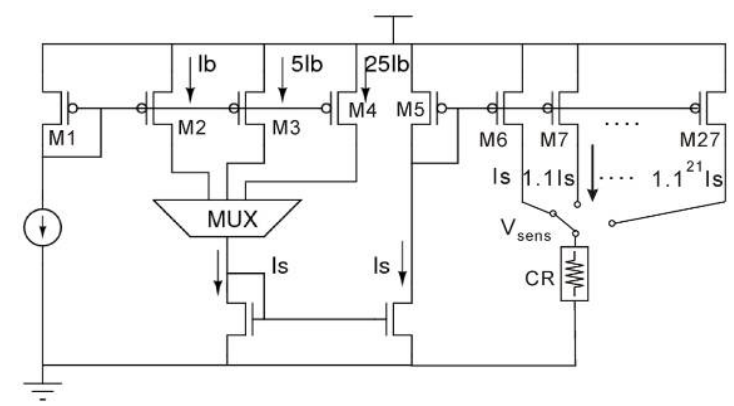

Fig. 3. Wide-range programmable exponential current bias schematic. 
control bits tune the current scaling in 22 exponential steps, from 1 to $1.1^{21}(=7.4)$. The higher two control bits provide coarse steps via programmable current gain with values of 1 , 5 and 25. The total tunable range of current is $60 \mathrm{nA}$ to $12.3 \mu \mathrm{A}$, which will keep $\mathrm{V}_{\mathrm{b}}$ between $0.6 \mathrm{~V}\left(\mathrm{~V}_{\min }\right)$ and $0.7 \mathrm{~V}$ $\left(\mathrm{V}_{\max }\right)$ for all values of $\mathrm{R}_{\mathrm{b}}$ between $60 \mathrm{k} \Omega$ to $15 \mathrm{M} \Omega$. In contrast, a linear current source with three segments would generate over three times the voltage input span, limiting the gain and resolution by a factor of three.

\section{B. Analog Memory}

To achieve high resolution measurement of the sensor's response, an analog memory (AM) is utilized to store the baseline output value $\left(\mathrm{V}_{\mathrm{b}}\right)$ and subsequently subtract this offset value during sensor readout. A simple calibration routine is used to set the value in the AM. When the sensor is idle $(\Delta \mathrm{R}=0)$, the readout circuit output should ideally be $0 \mathrm{~V}$. During calibration, a feedback loop from the amplifier output to the AM is used to drive the output to $0 \mathrm{~V}$. Calibration is complete once this state is achieved, and thus the AM will hold the proper baseline value. This approach also has the advantage of compensating for all non-ideal offsets in the base cancellation and gain blocks of Fig. 2.

The baseline resistances of MPN CRs are known to drift significantly within a matter of minutes. Ideally, the readout system should support a drift tracking re-calibration scheme. Our targeted MPN CRs are typically implemented within a micro gas chromatograph system [1] that periodically analyzes preconcentrated vapor samples and permits regular recalibration in a clear environment. Because the time between recalibration periods is on the order of minutes, charge leakage excludes the use of a capacitor-based AM. Therefore, the AM was implemented as a digital to analog converter (DAC) that can hold the AM value indefinitely. Note that after calibration, the AM-DAC holds the digital value of $R_{b}$ so it is readily available to determine $\Delta R / R_{b}$.

The disadvantage of a DAC-based AM is that it stores a discrete estimate of the baseline resistance. The AM output voltage can be expressed as

$$
V_{A M}=I_{R} R_{b}{ }^{\prime}
$$

where $R_{b}{ }^{\prime}$ is the DAC estimate of $R_{b} . I_{R} R_{b}{ }^{\prime}$ is within one LSB of the actual $I_{R} R_{b}$. To determine the necessary resolution of the $\mathrm{DAC}$, consider that the baseline cancellation error, $\varepsilon$, can be expressed by

$$
\varepsilon=I_{R}\left(R_{b}-R_{b}{ }^{\prime}\right) \leq \frac{V_{h}-V_{l}}{2^{N}}
$$

where $\mathrm{V}_{\mathrm{h}}$ to $\mathrm{V}_{1}$ is the output range of the DAC and $N$ is the DAC bit resolution. During calibration, the output never reaches exactly $0 \mathrm{~V}$ due to $\varepsilon$. Thus, if higher precision is required, $\varepsilon$ can be recorded during calibration and used in post processing the data. The primary restriction on $\varepsilon$ is that it should not exceed the input range of the amplifier. Because the programmable current bias already limits $V_{b}$ to
$0.6-0.7 \mathrm{~V}$, the DAC output range has been chosen to match this range. An 8-bit DAC resolution in this range allows 10bit resolution across the full output range. It was determined that an 8-bit R-2R ladder current-mode DAC limits the output error to less than $1 \mathrm{mV}$ and satisfies the requirement set by (6).

\section{Subtraction and Gain Block}

The subtraction and gain block was implemented using the fully differential amplifier shown in Fig. 4. The first stage is an operational transconductance amplifier (OTA), with $g_{m}=1 / R_{s}$. The second stage is a closed loop amplifier. The total gain is $A=g_{m}\left(R_{d}\right)=R_{d} / R_{s}$. Gain accuracy is ensured by careful resistance matching in the layout. During operation, the programmable gain is set to match the dynamic range of the sensor.

\section{Digital Block}

A digital control block is needed to configure the AM DAC inputs, programmable current bias, amplifier gain, and channel selection. A 6-byte memory was implemented along with a serial peripheral interface (SPI) communication block to minimize the number of $\mathrm{I} / \mathrm{O}$ pins required. As shown in Fig. 2, serial data are fed into the SPI and stored into memory registers to configure the settings for all analog circuits.

\section{RESULTS}

\section{A. Microsystem Implementation}

The new 8-channel CR-array readout circuit was fabricated in a $0.5 \mu \mathrm{m}$ CMOS process and consumes $66 \mu \mathrm{W}$ per channel with a $3.3 \mathrm{~V}$ supply. The die photograph of the

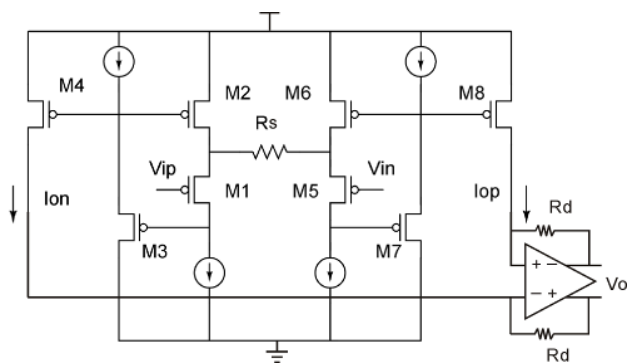

Fig. 4. Subtraction and gain block with a total gain of $R_{d} / R_{s}$.

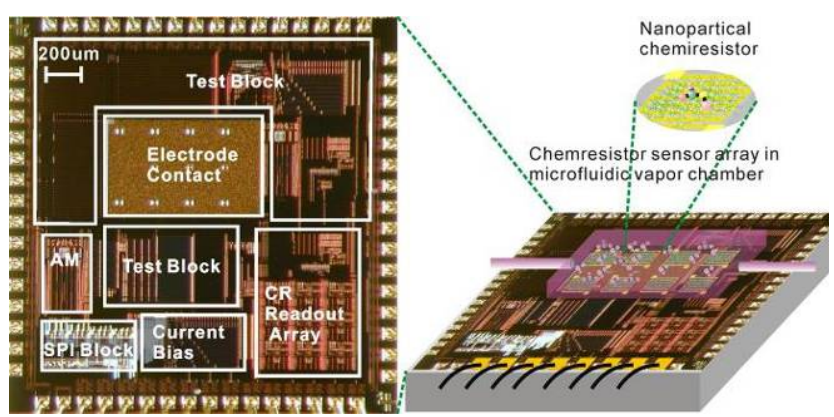

Fig.5. Die photograph of $2.2 \times 2.2 \mathrm{~mm} \mathrm{CR}$-array readout chip and illustration of the micro CR sensor system. 
$2.2 \times 2.2 \mathrm{~mm}$ chip is shown in Fig. 5. The electrode contact area permits direct connection between on-chip circuitry and interdigitated electrodes patterned on the chip's surface using e-beam lithography. To avoid damage to circuitry from post-CMOS e-beam patterning and sensor interface cross-linking, which was observed in previous iterations of the chip, the sensor array (electrode contact) area contains no circuitry. Metal 3 layer is placed beneath the sensor array area to create a planar surface that improves the reliability of surface routing. The sensor area is placed in the middle of the chip to facilitate post-wire-bonding application of a PDMS "cap" that creates a microfluidic vapor chamber on the surface of the chip. Fig. 5. shows an illustration of onCMOS microsystem concept, integrating a CR array and a microfluidic vapor chamber.

\section{B. Experimental results}

To verify baseline cancellation function of the CR readout chip, a test was performed with in a gas flow chamber using an external micro-array sensor device. Fig. 6 shows the output of CR readout circuit for OPH-MPN CR sensor responses to 2-butanone at different concentrations. The CR readout chip performs baseline cancellation, dramatically amplifying the resistance change as a result.

To characterize the dynamic range of the circuit, the baseline resistance was swept over a large range and the current bias was periodically re-programmed to minimize the $V_{b}$ range. Results show that $V_{b}$ can be held within the tight range of $0.61-0.72 \mathrm{~V}$, or values of $\mathrm{R}_{\mathrm{b}}$ from $52 \mathrm{k} \Omega$ to 13.28M $\Omega$. The $\Delta \mathrm{R} / \mathrm{R}$ resolution was characterized as $6 \sigma$ of the equivalent input noise of the chip over the baseline

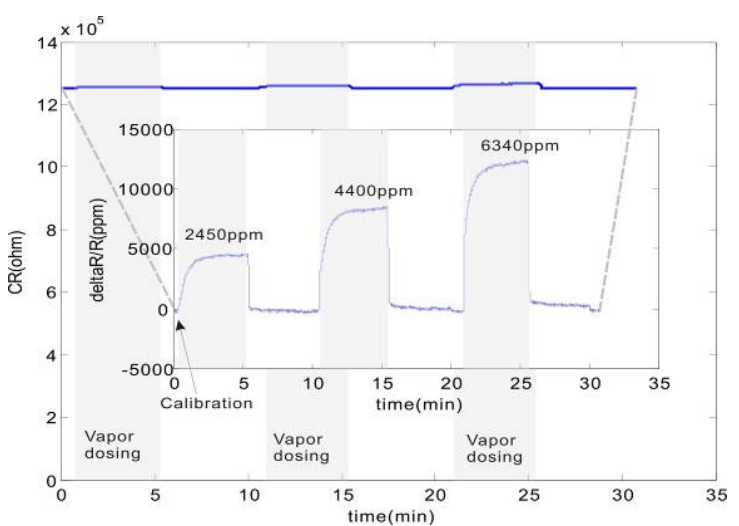

Fig.6. Output of CR readout circuit for OPH-MPN CR sensor responses to 2-butanone at different concentrations. CR readout circuit performs baseline cancellation, dramatically amplifying the resistance change.

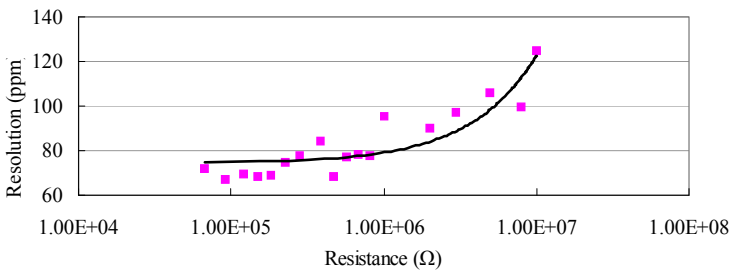

Fig.7. Chip readout resolution vs. baseline resistance. value. By sweeping resistance from $60 \mathrm{k} \Omega$ to $10 \mathrm{M} \Omega$, the chip readout resolution vs. resistance is plotted in Fig. 7. The plot shows the resolution is better than $125 \mathrm{ppm}$ over the resistance range, implying a $120 \mathrm{~dB}$ equivalent dynamic range.

\section{CONCLUSION}

This paper presented a compact, low-noise, baseline cancellation resistive readout circuit. The chip provides eight sensor readout channels, effectively cancels baseline variation over more than two orders of magnitude, provides drift tracking, and achieves $125 \mathrm{ppm}$ resolution in $\Delta R / R_{b}$ over whole baseline range, leading to an equivalent dynamic range of $120 \mathrm{~dB}$. The circuit was implemented in $0.5 \mu \mathrm{m}$ CMOS with a $3.3 \mathrm{~V}$ supply and consumes $66 \mu \mathrm{W}$ per channel. This circuit overcomes many of the instrumentation challenges presented by $\mathrm{CR}$ sensors and is well suitable for on-chip sensor array readout.

\section{REFERENCES}

[1] C. Lu, W. H. Steinecker, W. Tian, M. C. Oborny, J. M. Nichols, M. Agah, J. A. Potkay, H. K. L. Chan, J. Driscoll, R. D. Sacks, K. D. Wise, S. W. Pang, E. T. Zellers, "First-generation hybrid MEMS gas chromatograph," Lab on a Chip, vol. 5 (10), pp. 1123-1131, 2005.

[2] L. Han, X. Shi, W. Wu, F. L. Kirk, J. Luo, L. Wang, D. Mott, L. Cousineau, S. I. Lim, S. Lu, C. Zhong, "Nanoparticle-structured sensing array materials and pattern recognition for VOC detection," Sens. Actuators, B., vol. 106, pp. 431-441, 2005.

[3] W. H. Steinecker, M. P. Rowe, E. T. Zellers, "Model of vaporinduced resistivity changes in gold-thiolate monolayer-protected nanoparticle sensor films," Anal. Chem., vol. 79, pp. 4977-4986, 2007.

[4] Q. Zhong, R. A. Veeneman, W. H. Steinecker, C. Jia, S. A. Batterman, E. T. Zellers, "Rapid determination of ETS markers with a prototype field-portable GC employing a microsensor array detector," J. Environmental Monitoring, vol. 9, no. 5, pp. 440-448, 2007.

[5] M. Grassi, P. Malcovati, A. Baschirotto, "A 141-dB Dynamic Range CMOS Gas-Sensor Interface Circuit Without Calibration With 16-Bit Digital Output Word," IEEE J. Solid-State Circuits, vol. 42, no. 7, pp. 1543-1554, 2007.

[6] A. Flammini, D. Marioli, A. Taroni, "A low-cost interface to highvalue resistive sensors varying over a wide range," IEEE Trans. Instrumentation and Measurement, vol. 53, no. 4, pp. 1052-1056, 2004.

[7] D. Barrettino, M. Graf, S. Taschini, S. Hafizovic, C. Hagleitner, A. Hierlemann, "CMOS Monolithic Metal-Oxide Gas Sensor Microsystems," IEEE Sensors J., vol. 6, no. 2, pp. 276-286, 2006.

[8] C. Leung, D. Wilson, "Integrated interface circuits for chemiresistor arrays," Proc. IEEE Int. Symp. Circuits Syst., vol. 5, pp. 5914-5917, 2005.

[9] T. J. Koickal, A. Hamilton, S. L. Tan, J. A. Covington, J. W. Gardner, T. C. Pearce, "Analog VLSI Circuit Implementation of an Adaptive Neuromorphic Olfaction Chip," IEEE Trans. on Circuits and Systems I, vol. 54, pp. 60-73, 2007.

[10] M. Grassi, P. Malcovati, A. Baschirotto, "A 160 dB Equivalent Dynamic Range Auto-Scaling Interface for Resistive Gas Sensors Arrays," IEEE J. Solid-State Circuits, vol. 42, no. 3, pp. 518 -528, 2007

[11] D. Rairigh, G. Warnell, C. Xu, E. T. Zellers, A. J. Mason, "CMOS Baseline Tracking and Cancellation Instrumentation for NanoparticalCoated Chemiresistors," IEEE Trans. Biomedical Circ. Systems, vol. 3 (5), pp. 267-276, 2009. 